\title{
Galanin receptor 2 utilizes distinct signaling pathways to suppress cell proliferation and induce apoptosis in HNSCC
}

\author{
TAKEHARU KANAZAWA ${ }^{1}$, KIYOSHI MISAWA ${ }^{2}$, YUKI MISAWA ${ }^{2}$, MIKIKO MARUTA ${ }^{1}$, TAKAYUKI UEHARA ${ }^{3}$, \\ KAZUMI KAWADA $^{1}$, TAKAFUMI NAGATOMO ${ }^{1}$ and KEIICHI ICHIMURA ${ }^{1}$ \\ ${ }^{1}$ Department of Otolaryngology-Head and Neck Surgery, Jichi Medical University School of Medicine, Shimotsuke, \\ Tochigi 329-0498; ${ }^{2}$ Department of Otolaryngology-Head and Neck Surgery, Hamamatsu University School of Medicine, \\ Hamamatsu, Shizuoka 431-3192; ${ }^{3}$ Department of Otorhinolaryngology-Head and Neck Surgery, \\ Graduate School of Medicine, University of the Ryukyus, Nishihara, Okinawa 903-0215, Japan
}

Received September 23, 2013; Accepted April 4, 2014

DOI: $10.3892 / \mathrm{mmr} .2014 .2362$

\begin{abstract}
Galanin and its receptors, GALR1 and GALR2, are tumor suppressors and represent therapeutic targets in head and neck squamous cell carcinoma (HNSCC). In the present study, it was demonstrated that the re-expression of GALR1 in GALR1 and GALR2-negative HNSCC cells suppresses tumor cell proliferation. This is mediated via extracellular-regulated protein kinase-1/2 (ERK1/2)-dependent effects on the cyclin-dependent kinase inhibitors (CKI) and cyclin D1. In combination with galanin, GALR2 also suppressed proliferation by increasing CKI and decreasing cyclin D1 levels. In contrast to GALR1, overexpression of GALR2 also induced caspase-3-dependent apoptosis. It was identified that in GALR2-transfected cells, galanin induced activation of ERK1/2 and suppressed cell proliferation. Galanin stimulation also decreased the expression of cyclin D1 and induced apoptotic DNA ladder formation in GALR2-transfected cells. Pretreatment with the ERK1/2-specific inhibitor U0126 and pertussis toxin prevented the suppression of cyclin D1 expression, however did not affect DNA ladder formation. In conclusion, GALR2 expression in the presence of galanin exerts antitumor effects via cell cycle arrest and apoptotic pathways, and reactivation of these pathways may have therapeutic benefits in HNSCC.
\end{abstract}

\section{Introduction}

Despite the development and use of aggressive therapeutic techniques, the prognosis for patients with advanced head and

Correspondence to: Dr Takeharu Kanazawa, Department of Otolaryngology, Head and Neck Surgery, Jichi Medical University School of Medicine, 3311-1 Yakushiji, Shimotsuke, Tochigi 329-0498, Japan

E-mail: kanatake@omiya.jichi.ac.jp

Key words: G protein-coupled receptors, extracellular-regulated protein kinase-1/2, cell cycle arrest, head and neck neoplasm neck squamous cell carcinoma (HNSCC) remains poor $(1,2)$. However, genomic and proteomic studies are beginning to provide insight into the molecular drivers of such cancer types, which may aid the design of targeted therapeutic agents. At present, one-third of pharmaceuticals on the market were reported to exert their therapeutic effect by interacting with a G-protein coupled receptor (GPCR) (3). Although GPCRs control a wide array of signaling pathway in all tissues, the full range of their effects remains to be elucidated (4). Galanin is a 30-amino-acid peptide and its receptors, GALR1, GALR2 and GALR3, are members of the GPCR superfamily. It is known that these receptors are variably expressed in numerous normal tissues, including squamous epithelium and certain types of tumor, including glioblastoma, neuroblastoma, small cell lung cancer and HNSCC (5-8). The effects of galanin signaling are variously reported in different tumor types. Seufferlein et al (9) reported that galanin has mitogenic effects in small cell lung carcinoma, whereas, Iishi et al (10) reported that galanin inhibits pancreatic carcinogenesis. With the development of functional analysis methods for individual receptors, it became evident that galanin, GALR1 and GALR2 may act as tumor suppressors $(8,11-15)$. Our previous study revealed that the activation of the GALR1 signaling pathway suppressed tumor cell proliferation via phosphorylation of extracellular signal regulated kinase 1/2 (ERK1/2), which was associated with the upregulation of cyclin-dependent kinase inhibitors (CKIs) and the downregulation of cyclin D1 in HNSCC (8). Other studies revealed that GALR1 inhibits proliferation by inactivating the mitogen-activated protein kinase (MAPK) pathway in oral squamous cell carcinoma (16).

Activation of the GALR2 signaling pathway suppresses cell proliferation through the induction of apoptosis in a number of tumor types, including $\operatorname{HNSCC}(6,12,17)$ and this may proceed via several distinct underlying mechanisms. The apoptotic effects of the GALR2 signaling pathway are partially mediated by dephosphorylation of Akt and the subsequent activation of the proapoptotic Bcl-2 family protein, Bad, in PC12 cells, a pheochromocytoma cell line (17). Our recent study using an adeno-associated virus vector, demonstrated that the reduction of HNSCC in response to GALR2 expression in the presence of galanin was due to the induction of apop- 
tosis rather than cell cycle arrest. GALR2-mediated apoptosis was caspase-independent and involved the downregulation of ERK1/2 and the induction of the pro-apoptotic Bcl-2 protein, Bim. Despite this insight, the signaling pathways modulated by GALR2 in HNSCC are not fully understood and more information is required in order to translate the above findings into clinical applications. Therefore, the aim of the present study was to further delineate in vitro the GLAR2-dependent signaling pathways that induce either cell cycle arrest and/or apoptosis in a HNSCC cell line.

\section{Materials and methods}

Cell culture and proliferation assay. GALR2-expressing HNSCC cells were established as previously described (12). The C-terminal HA-tagged GALR2 sequence was obtained from a human cDNA library (Invitrogen Life Technologies, Carlsbad, CA, USA) and subcloned into a pcDNA3 vector (Invitrogen Life Technologies) containing an internal ribosomal entry site (Ires) and green fluorescent protein (GFP) sequence. The pCMVIresGFP was used as a negative control. The UM-SCC-1-GALR2 and UM-SCC-1-mock cells were established by transfecting pCMVGALR2IresGFP or pCMVIresGFP into the human oral carcinoma cell line, UM-SCC-1, using lipofectamine regular reagents (Invitrogen Life Technologies). GFP-positive cells were selected by flow cytometry using a FACS Vantage SE (BD Biosciences, San Diego, CA, USA). The cells were cultured in Dulbecco's Modified Eagle's Medium (DMEM; Gibco, Grand Island, NY, USA) supplemented with $10 \%$ heat-inactivated fetal bovine serum, $100 \mathrm{U} / \mathrm{ml}$ penicillin and $100 \mu \mathrm{g} / \mathrm{ml}$ streptomycin (Irvine Scientific, Santa Ana, CA, USA) at $37^{\circ} \mathrm{C}$ in $5 \% \mathrm{CO}_{2}$. To examine cell proliferation and morphology, $24 \mathrm{~h}$ following cell plating, they were cultured with serum-free media (SFM) containing $0.1 \%$ BSA for $24 \mathrm{~h}$ to induce quiescence. Then, $0.5 \mu \mathrm{M}$ of galanin (AnaSpec, San Jose, CA, USA) was added. The ERK1/2 inhibitor U0126 (Cell Signaling Technology, Inc., Beverly, MA, USA) was added $1 \mathrm{~h}$ prior to galanin treatment and pertussis toxin (PTX; Sigma, St. Louis, MO, USA) was added $10 \mathrm{~h}$ prior to treatment. Cell proliferation was determined by counting the cells with a Coulter counter model Z1 (Beckman Coulter Inc., Hialeah, FL, USA). To observe changes in cell morphology, images captured by the Olympus IX71 inverted microscope were taken (Olympus Corporation, Tokyo, Japan). The present study was approved by the ethics committee of Jichi Medical University (Shimotsuke, Tochigi, Japan).

Immunocytochemistry. The cells were seeded on coverslips. Following $24 \mathrm{~h}$ of incubation, the cells were fixed with $4 \%$ paraformaldehyde and then stained with mouse monoclonal anti-HA tag antibody (Covance, Berkeley, CA, USA) and Hoechst 33342 (Molecular Probes, Leiden, Netherlands). Following incubation with AlexaFluor 546 goat anti-mouse IgG1 (Molecular Probes), the localization of exogenous GALR1 was determined using an Olympus FV-500 confocal microscope (Olympus Corporation).

Immunoblotting. The cells were lysed with $1 \%$ Nonidet P-40 lysis buffer containing protease inhibitors (Calbiochem, La Jolla, CA, USA). The supernatant was collected and the protein content was measured using the Bio-Rad protein assay (Bio-Rad, Richmond, CA, USA). Equal amounts of protein were electrophoresed on $10 \%$ SDS-PAGE gels and transferred to Hybond-P (Amersham Biosciences UK Ltd, Little Chalfont, Buckinghamshire, England). The membranes were incubated overnight with primary antibody at $4^{\circ} \mathrm{C}$, followed by incubation with anti-mouse secondary antibody horseradish peroxidase conjugate (Amersham Biosciences UK Ltd) and detected by chemiluminescence and autoradiography using a Hyperfilm obtained from Kodak (Rochester, NY, USA). ERK1/2 activation was evaluated with rabbit polyclonal phoshpo-ERK1/2 antibody and total ERK1/2 antibody (Cell Signaling Technology, Inc.). Expression of cyclin D1 was detected using a mouse monoclonal antibody (DakoCytomation Norden A/S, Glostrup, Denmark). Cytochrome $\mathrm{C}$ oxidase subunit 4 (COX4) was detected using a mouse monoclonal anti-COX4 antibody (Abcam plc. Cambridge, UK) as an internal control.

Apoptosis analysis. Induction of apoptosis was evaluated by detection of DNA ladder formation. Serum-starved cells were treated with galanin for $24 \mathrm{~h}$, genomic DNA was isolated using the DNeasy tissue kit (Qiagen, Valencia, CA, USA) and then loaded onto a $2 \%$ agarose gel. The DNA ladders stained with ethidium bromide were visualized under UV light.

Statistical analysis. The results were examined for statistical significance by using the Mann-Whitney $\mathrm{U}$ test $\left({ }^{* *} \mathrm{P}<0.01\right)$.

\section{Results}

Exogenous GALR2 expression. To assess the expression and cellular localization of exogenous GALR2, cells grown on coverslips were stained with an HA-tag antibody. Immunofluorescence revealed that all the GFP-positive cells exhibited exogenous GALR2 expression localized to the cytoplasmic membrane, as expected for a GPCR (Fig. 1). The expression of GALR2 in the parental UM-SCC-1 cells was undetectable and less than that in normal human tissue (data not presented). Therefore, the differences in the results that we obtained with the UM-SCC-1-GALR2 and UM-SCC-1-mock cells following galanin stimulation should reflect mainly the function of GALR2.

Galanin and GALR2 induce ERK1/2 activation and inhibit cell proliferation. We next examined whether the GALR2/galanin signaling pathway involved activation of ERK1/2. As demonstrated in Fig. 2A, ERK1/2 activation was observed in UM-SCC-1-GALR1 at all time points following stimulation. By contrast, ERK1/2 phosphorylation in UM-SCC-1 cells was not observed at any of the time points (Fig. 2A). To determine the role of GALR2 in proliferation, cells starved for $24 \mathrm{~h}$ were treated with $0.5 \mu \mathrm{M}$ of galanin and counted with a Coulter counter. Fig. 2B reveals the relative proliferation of UM-SCC-1-GALR2 and UM-SCC-1-mock cells following galanin treatment. Proliferation of UM-SCC-1-GALR2 cells was reduced by $32 \%$ compared with the control UM-SCC-1-mock cells when treated with galanin $(\mathrm{P}<0.01)$.

U0126 and PTX abrogates galanin-and GALR2-dependent activation of ERK1/2. Since GALR2 activates ERK1/2 and 

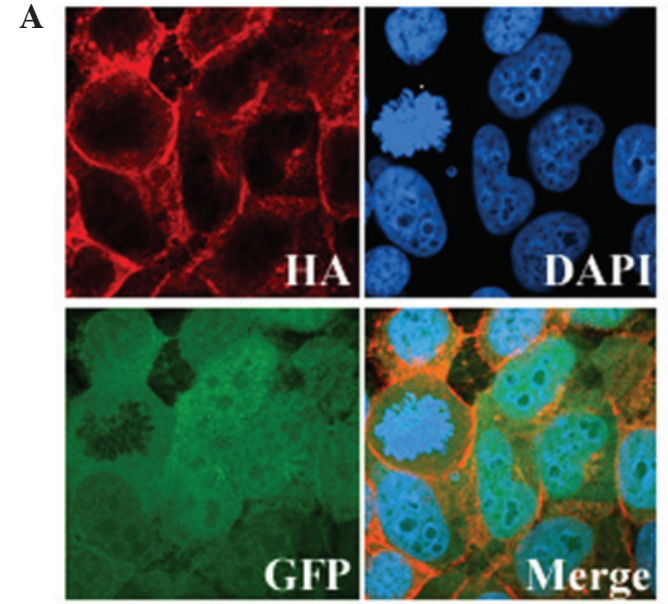

B
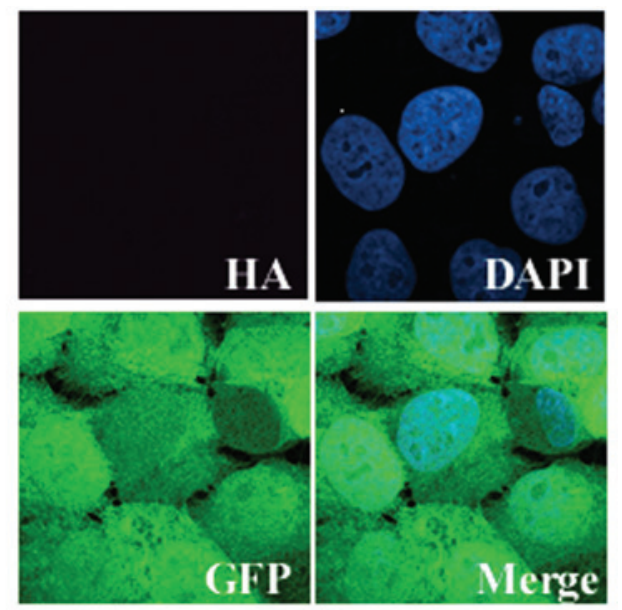

Figure 1. GALR2 expression in GALR2HA-transfected UM-SCC-1 cells. (A) GALR2HA-transfected cells; (B) mock-transfected cells. Images demonstrate HA-tag (upper left), Hoechst 33342 (upper right), GFP (lower left) and merge (lower right). GALR2, galanin receptor 2; GFP, green fluorescent protein.

A

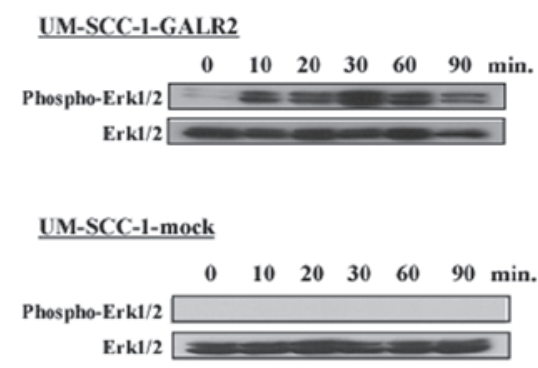

B

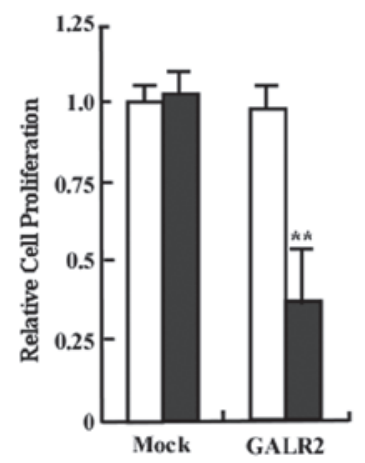

Figure 2. Effect of galanin stimulation on ERK1/2 activation and cell proliferation. (A) Immunoblot revealing galanin-induced ERK1/2 activation. (B) Relative cell proliferation following $0.5 \mu \mathrm{M}$ galanin treatment. Closed bars indicate galanin treatment and open bars indicate no treatment. Proliferation of UM-SCC-1-GALR2 cells was significantly reduced compared with the control UM-SCC-1-mock cells when treated with galanin (**P<0.01). ERK1/2, extracellular-regulated protein kinase 1/2; GALR2, galanin receptor 2.

inhibits cell proliferation, we investigated whether these two events were functionally associated. Treatment with either U0126 (an ERK inhibitor) or PTX (a Gai pathway inhibitor) abrogated galanin- and GALR2-mediated ERK1/2 activation. Together these results indicate that GALR2-induced ERK1/2 activation may proceed through the same signaling pathway activated by GALR2 (Fig. 3).

U0126 partially abrogates the galanin- and GALR2 induced anti-proliferative effect. Our previous study demonstrated that galanin suppressed cell proliferation and induced apoptosis in HNSCC cells. To determine the role of ERK1/2 in GALR2-induced arrest, cells were cultured in the presence or absence of U0126 and then stimulated with galanin. As demonstrated in Fig. 4A, galanin-treated UM-SCC-1-GALR2 cells were significantly decreased compared with untreated or UM-SCC-1-mock cells. U0126 pretreatment abrogated the galanin- and GALR2-induced anti-proliferative effect; however the effect was only partial (48\% reduction). Fig. 4B reveals the striking morphological changes in galanin-treated UM-SCC-1-GALR2 cells, which were partially abrogated by pretreatment with U0126. These results suggested that

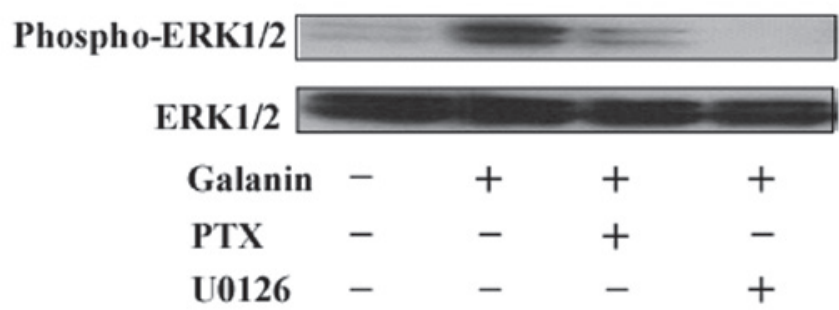

Figure 3. The Gai pathway leads to ERK1/2 activation. Serum-starved UM-SCC-1 cells were pretreated with U0126 and PTX prior to galanin treatment, and evaluated for ERK1/2 activation. ERK1/2, extracellular-regulated protein kinase $1 / 2$; PTX, pertussis toxin.

while ERK1/2 is a critical mediator of the GALR2-induced anti-proliferative effect, additional mechanisms co-exist.

GALR2-dependent activation of ERK1/2 is associated with cyclin D1 regulation but not with induction of apoptosis. To further investigate whether apoptosis or cell cycle arrest was associated with the ERK1/2 pathway, DNA ladder formation and cyclin D1 expression were examined in galanin-treated 
A

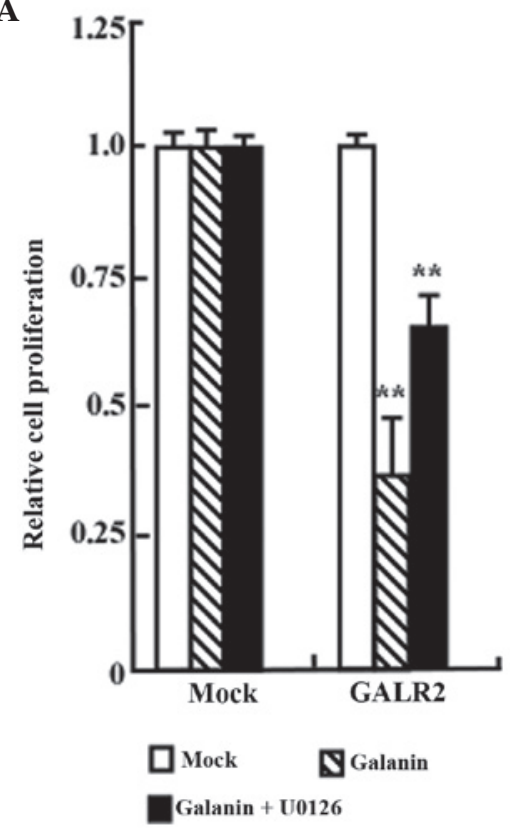

B

GALR2

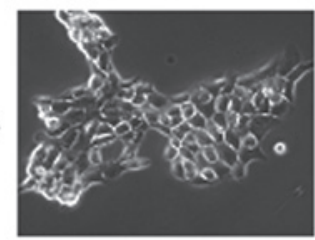

GALR2 + galanin

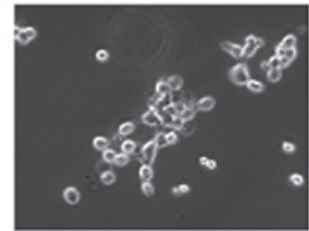

GALR2 + galanin $+\mathrm{U} 0126$

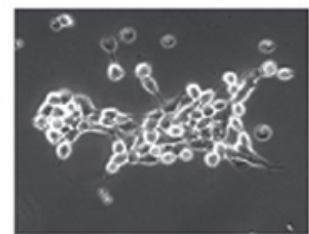

Figure 4. Effect of galanin and U0126 on cell proliferation and morphological appearance. (A) Serum-starved UM-SCC-1-GALR1 cells were pretreated with U0126 prior to the addition of galanin. A total of $24 \mathrm{~h}$ later, the cells were counted (** $\mathrm{P}<0.01)$ and $(\mathrm{B})$ the images were captured. Galanin-treated UM-SCC-1-GALR2 cells were significantly decreased compared with untreated or UM-SCC-1-mock cells $\left({ }^{* *} \mathrm{P}<0.01\right)$. U0126 pretreatment significantly abrogated the galanin- and GALR2-induced anti-proliferative effect $(\stackrel{* *}{*}<0.01)$; however the effect was only partial. GALR2, galanin receptor 2.

\section{A}

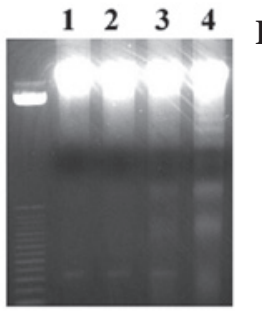

B

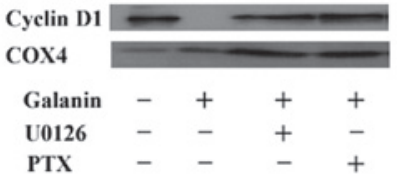

1. GALR2

2. mock+Galanin

3. GALR2+Galanin

4. GALR2+Galanin+U0126

Figure 5. Effect of galanin and specific cell signaling inhibitors on apoptotic DNA ladder formation and cyclin D1 expression. (A) Apoptotic DNA ladder formation; (B) western blot for cyclin D1. GALR2, galanin receptor 2.

UM-SCC-1-GALR2 cells with or without U0126 pretreatment. As demonstrated in Fig. 5A, galanin-treated UM-SCC-1-GALR2 cells exhibited DNA ladder formation that was not abrogated by U0126. Galanin and GALR2 expression lead to a reduction in cyclin D1 expression, similar to our previous study, and both U0126 and PTX abrogated cyclin D1 inhibition completely (Fig. 5B). Therefore, GALR2-induced ERK1/2 expression appears to be associated with cell cycle arrest via the Gai pathway, rather than with apoptosis.

\section{Discussion}

In a previous study, we demonstrated the function of the GALR1 and GALR2 signaling pathways (8,12-15). In HNSCC cell lines in which GALR1 and GALR2 expression were silenced, it was demonstrated that stable re-expression of GALR1 suppressed proliferation via ERK1/2-mediated effects on cyclin-dependent kinase inhibitors and cyclin D1 (8). By contrast, in GALR2-transfected HNSCC cells, galanin suppressed proliferation, however also induced apoptosis (12). The loss or inactivation of the galanin-GALR signaling cascade provides tumor cells with another mechanism of avoiding cell death. Therefore, it was deduced that GALR1 and GALR2 function as tumor suppressors and therefore, these proteins became the focus as therapeutic targets for HNSCC. GALR2-induced cell cycle arrest is associated with the upregulation of p27kip1 and p57kip2, and the downregulation of cyclin D1, while the apoptosis induced by GALR 2 is partially caspase- 3 dependent (12). Previously, we published two studies that examined the role of GALR2 in HNSCC $(11,18)$. First, it was demonstrated that the expression of GALR2 mRNA is lost in HNSCC as a consequence of DNA methylation and that this may be a critical event during the development of HNSCC (11). Secondly, it was identified that adeno-associated vector delivery of GALR2 induced apoptosis that was dependent on induction of the pro-apoptotic Bcl-2 protein, Bim (18). Together, these results indicated that activation of GALR2 signaling may be of therapeutic benefit. However, the downstream signaling pathways that govern the effects of GALR2 have remained poorly understood.

In the present study, it was demonstrated that while GALR1 only induces cell cycle arrest, GALR2 induces both cell cycle arrest and apoptosis. These findings suggest that there are bifurcations in the signaling pathways downstream of GALR2. GALR2 signals through multiple classes of $\mathrm{G}$ proteins and stimulates multiple intracellular pathways $(7,19)$. The most commonly reported pathway involves phospholipase C (PLC) activation, which increases inositol phosphate hydrolysis, triggers the release of $\mathrm{Ca}^{2+}$ into the cytoplasm and leads to the opening of $\mathrm{Ca}^{2+}$-dependent chloride channels (19-22). These GALR2-mediated intracellular effects 
are not affected by PTX, demonstrating that GALR2 may act through Gq/11-types of G proteins (19). However, suggestions that GALR2 interacts with other types of G protein are often controversial. Fathi et al, reported that galanin-dependent cAMP production was observed in HEK-293 cells (23). Similar to GALR1, this inhibition was PTX-sensitive, which suggested a coupling of GALR2 to the inhibition of adenylate cyclase through Gai-type $\mathrm{G}$ proteins $(23,24)$. As described above, GALR2 initiates multiple signaling pathways and the PTX-sensitive Gai pathway is common to GALR1 and GALR2, and it was therefore hypothesized that this pathway is important for the anti-proliferative effect of GALRs. Indeed, in the present study, U0126 abrogated ERK1/2 activation and prevented the downregulation of cyclin D1 and inhibition of cell proliferation caused by GALR expression. Similarly, PTX also abrogated ERK1/2-dependent downregulation of cyclin D1. Therefore, GALR2-induced ERK1/2 activation and cell cycle arrest associated with cyclin D1 expression are closely associated, and it appears that ERK1/2 is activated by the Gai pathway in the same manner as GALR1 signaling (8). However, inhibition of ERK1/2 did not abrogate galanin- and GALR2-induced apoptotic DNA ladder formation, indicating that GALR2 utilizes different signaling pathways in order to induce apoptosis.

Although our studies and others have demonstrated that GALR2 induces apoptosis, it appears that different mechanisms may underlie the GALR2-mediated anti-proliferative effect. Berger et al suggested that GALR2-induced apoptosis was in part due to the activation of caspase-3 and DNA fragmentation (6). However, a caspase-3 inhibitor was unable to block the appearance of apoptotic morphology and did not rescue cell viability. Therefore, they concluded that caspase-3 is not an obligatory mediator of apoptosis triggered by the activation of GALR2 in neuroblastoma cells, as was observed for rotenone-induced apoptosis (6). Tofighi et al have provided an alternative mechanism of GALR2-induced apoptosis (17). The authors suggest that GALR2 blocks activation of the pro-survival Akt kinase, which leads to a net dephosphorylation of the apoptotic Bad protein and consequent caspase-3 dependent cell death (17). Our previous study demonstrated that GALR2 triggers caspase-3-dependent apoptosis and cell cycle arrest in p53 mutant HNSCC cells (12). Recently, we have also revealed that GALR2-mediated apoptosis may also occur in a caspase-independent manner, which involves the downregulation of ERK1/2, followed by the induction of the pro-apoptotic Bcl-2 protein, Bim (18). In that particular study, a Bim-independent pathway for apoptosis was also observed (18). These findings suggest that GALR2 employs diverse and complex pathways in order to induce apoptosis.

In the present study, GALR2 activated ERK1/2 and this effect was associated with anti-proliferative effects. Conversely, the expression of GALR2 from rAAV vectors led to a significant downregulation of ERK1/2 (18). Although the reasons for this discrepancy are unclear, we note that similar paradoxical effects are also observed in GALR1 signaling. For example, Henson et al, reported that the antiproliferative effects of GALR1 are via ERK1/2 inhibition, whereas we demonstrated that GALR1 required ERK1/2 activation in order to induce arrest $(8,16)$. This 'Janus-like' activity may be a general feature of certain GPCRs. For example, soma- tostatin receptors (SSTRs) may either inhibit or activate Akt and ERK, depending on the precise physiological context (25). Additionally, heterodimeric SSTRs stimulate ERK activity in the presence of high ligand concentrations, however inhibit ERK at low concentrations (26).

Wittau et al demonstrated that there are multiple signaling pathways downstream of GALR2 in small cell lung cancer cells, and that this is due to the specific coupling of GALR2 to various G-proteins (7). This evidence supports our hypothesis that GALR2 utilizes multiple signaling pathways to mediate its antiproliferative effect, and that ERK activation is associated with the robustness of cell cycle arrest following activation of GALR2 signaling. In conclusion, GALR2 activation, or stimulation of the associated downstream signaling cascades, may be an attractive therapeutic strategy in the treatment of HSCC.

\section{Acknowledgements}

This study was supported by a Grant-in-Aid for Scientific Research (no. 22591916 and 23592539) from the Ministry of Education, Culture, Sports, Science, and Technology of Japan.

\section{References}

1. Kamangar F, Dores GM and Anderson WF: Patterns of cancer incidence, mortality, and prevalence across five continents: defining priorities to reduce cancer disparities in different geographic regions of the world. J Clin Oncol 24: 2137-2150, 2006 .

2. Shibuya K, Mathers CD, Boschi-Pinto C, Lopez AD and Murray CJ: Global and regional estimates of cancer mortality and incidence by site: II. Results for the global burden of disease 2000. BMC cancer 2: 37, 2002.

3. DeWire SM, Violin JD: Biased ligands for better cardiovascular drugs: dissecting G-protein-coupled receptor pharmacology. Circ Res 109: 205-216, 2011.

4. Hill SJ: G-protein-coupled receptors: past, present and future. Br J Pharmacol 147 (Suppl 1): S27-S37, 2006.

5. Berger A, Santic R, Almer D, et al: Galanin and galanin receptors in human gliomas. Acta Neuropathol 105: 555-560, 2003.

6. Berger A, Lang R, Moritz K, et al: Galanin receptor subtype GalR2 mediates apoptosis in SH-SY5Y neuroblastoma cells. Endocrinology 145: 500-507, 2004.

7. Wittau N, Grosse R, Kalkbrenner F, Gohla A, Schultz G and Gudermann T: The galanin receptor type 2 initiates multiple signaling pathways in small cell lung cancer cells by coupling to $\mathrm{G}(\mathrm{q}), \mathrm{G}(\mathrm{i})$ and $\mathrm{G}(12)$ proteins. Oncogene 19: 4199-4209, 2000.

8. Kanazawa $\mathrm{T}$, Iwashita $\mathrm{T}$, Kommareddi $\mathrm{P}$, et al: Galanin and galanin receptor type 1 suppress proliferation in squamous carcinoma cells: activation of the extracellular signal regulated kinase pathway and induction of cyclin-dependent kinase inhibitors. Oncogene 26: 5762-5771, 2007.

9. Seufferlein $\mathrm{T}$ and Rozengurt E: Galanin, neurotensin, and phorbol esters rapidly stimulate activation of mitogen-activated protein kinase in small cell lung cancer cells. Cancer Res 56: 5758-5764, 1996.

10. Iishi H, Tatsuta M, Baba M, et al: Inhibition by galanin of experimental carcinogenesis induced by azaserine in rat pancreas. Int J Cancer 75: 396-399, 1998.

11. Misawa Y, Misawa K, Kanazawa T, et al: Tumor suppressor activity and inactivation of galanin receptor type 2 by aberrant promoter methylation in head and neck cancer. Cancer 120 , 205-213, 2014.

12. Kanazawa T, Kommareddi PK, Iwashita T, et al: Galanin receptor subtype 2 suppresses cell proliferation and induces apoptosis in p53 mutant head and neck cancer cells. Clin Cancer Res 15: 2222-2230, 2009.

13. Kanazawa $\mathrm{T}$, Misawa $\mathrm{K}$ and Carey TE: Galanin receptor subtypes 1 and 2 as therapeutic targets in head and neck squamous cell carcinoma. Expert Opin Ther Targets 14: 289-302, 2010 
14. Misawa K, Kanazawa T, Misawa Y, et al: Galanin has tumor suppressor activity and is frequently inactivated by aberrant promoter methylation in head and neck cancer. Transl Oncol 6: 338-346, 2013.

15. Misawa K, Ueda Y, Kanazawa T, et al: Epigenetic inactivation of galanin receptor 1 in head and neck cancer. Clin Cancer Res 14: 7604-7613, 2008.

16. Henson BS, Neubig RR, Jang I, et al: Galanin receptor 1 has anti-proliferative effects in oral squamous cell carcinoma. J Biol Chem 280: 22564-22571, 2005.

17. Tofighi R, Joseph B, Xia S, et al: Galanin decreases proliferation of PC12 cells and induces apoptosis via its subtype 2 receptor (GalR2). Proc Natl Acad Sci USA 105: 2717-2722, 2008.

18. Uehara T, Kanazawa T, Mizukami H, et al: Novel anti-tumor mechanism of galanin receptor type 2 in head and neck squamous cell carcinoma cells. Cancer Sci: Oct 30, 2013 (Epub ahead of print).

19. Lang R, Gundlach AL and Kofler B: The galanin peptide family: receptor pharmacology, pleiotropic biological actions, and implications in health and disease. Pharmacol Ther 115: 177-207, 2007.

20. Fathi Z, Cunningham AM, Iben LG, et al: Cloning, pharmacological characterization and distribution of a novel galanin receptor. Brain Res Mol Brain Bres 51: 49-59, 1997.
21. Smith KE, Forray C, Walker MW, et al: Expression cloning of a rat hypothalamic galanin receptor coupled to phosphoinositide turnover. J Biol Chem 272: 24612-24616, 1997.

22. Wang S, Clemmons A, Strader C and Bayne M: Evidence for hydrophobic interaction between galanin and the GalR1 galanin receptor and GalR1-mediated ligand internalization: fluorescent probing with a fluorescein-galanin. Biochemistry 37: 9528-9535, 1998.

23. Fathi Z, Battaglino PM, Iben LG, et al: Molecular characterization, pharmacological properties and chromosomal localization of the human GALR2 galanin receptor. Brain Res Mole Brain Res 58: 156-169, 1998.

24. Wang S, Hashemi T, He C, Strader C and Bayne M: Molecular cloning and pharmacological characterization of a new galanin receptor subtype. Mol Pharmacol 52: 337-343, 1997.

25. Hagemeister AL, Kittilson JD, Bergan HE and Sheridan MA: Rainbow trout somatostatin receptor subtypes SSTR1A, SSTR1B, and SSTR2 differentially activate the extracellular signal-regulated kinase and phosphatidylinositol 3-kinase signaling pathways in transfected cells. J Mol Endocrinol 45: 317-327, 2010.

26. War SA and Kumar U: Coexpression of human somatostatin receptor-2 (SSTR2) and SSTR3 modulates antiproliferative signaling and apoptosis. J Mol Signal 7: 5, 2012. 